$\xi=$ 离

\title{
Reducing the risk to marine ports from invasive mosquito species, zika, dengue, chikungunya viruses and filariasis
}

\author{
Thomas M. Kollars Jr. ${ }^{1 *}$, Peggy G. Kollars ${ }^{2}$, Bannie Hulsey ${ }^{3}$ \\ ${ }^{1}$ College of Health Sciences, Liberty University, Lynchburg, Virginia, USA; previous address \\ Center for Science and Technology Advancement, Washington DC, USA \\ ${ }^{2}$ MEVLABS, Statesboro, Georgia, USA \\ ${ }^{3}$ Crisp Academy, Cordele, Georgia, USA \\ *Corresponding authorE-mail: tkollars@ liberty.edu
}

\begin{abstract}
Background: Mosquitoes are the principal vector of pathogens to humans throughout the world. Ports are a primary means to which invasive mosquito species and the pathogens they carry are introduced into new areas and where the mosquito species was once eradicated.

Methods: The ports of Porto Cortes (Honduras) and Savannah Port, (USA) were evaluated using the Bioagent Transport and Environmental Modeling System (BioTEMS) invasive species model to determine what species would be most likely to invade and survive in the Jebel Ali Port area (UAE). The Porto Cortes study site was evaluated to determine if mosquito control using ecofriendly pesticides was practical at the port of origin.

Conclusion: The Bioagent Transport and Environmental Modeling System TIGER model provides information that can be utilized to interdict and control invasive mosquito species to reduce the risk they become permanently established. The receiving ports were at risk from mosquito invasion, however, because of niche parameters, the Savannah Port was at risk from four invasive species from Honduras and the Jebel Ali Port was at risk from Ae. albopictus from Porto Cortes and Savannah and Ae. vexans from Savannah. Mosquito control was obtained at the point of origin and optimal surveillance and control sites were identified.
\end{abstract}

Keywords: Aedes Albopictus; Arbovirus; Invasive Species; Port Security; Zika.

\section{Introduction}

Globally, mosquitoes are the principle cause of vector-borne diseases in humans, causing millions of deaths and hundreds of millions of patient-doctor visits. Preventive medicine is critical in reducing morbidity and mortality from infectious diseases. Preventive mosquito intervention includes, mosquito control campaigns, personal protection with clothing and the use of repellents, and indoor and outdoor mosquito pesticide treatment. The United Arab Emirates has had a long history of successful integrated mosquito management campaign resulting from anti-malaria efforts (Dar et al. 1993). This effort continues, for example, Dr. Juma Bilal Fairuz, the Director of Preventive Medicine at the Ministry of Health (UAE) stated in regards to dengue, "the country's long history in battling malaria, another mosquito-borne disease, had protected its population from local transmission" (IPS 2006). Zika virus and several other viruses are spreading rapidly on a global scale. Several mosquito species are capable of invading new geographic regions and exploiting niches that are similar to their natural home ranges. These mosquitoes, and the pathogens they carry, may pose significant medical and veterinary health risks in naïve populations. The principle factor responsible for the introduction of disease vectors through marine invasions are air and ship transport (Lodge 2006, Tatem et al. 2006, Meyerson \& Mooney (2007).

Both abiotic and biotic factors affect the survival of the invading species, including micro-habitat, temperature, rainfall, breeding sites, host similarities, reinvasion and inter-species competition. Continual evaluation of the risk of importation of pathogens and vectors is required both to prevent disease outbreaks and to maintain uninterrupted trade. International trade with container ships is important economically between Porto Cortez (Honduras), Savannah (United States of America) and Dubai (United Arab Emirates). The current paper discusses the potential of several mosquito species originating from Central America to invade into the ports of Jebel Ali, Dubai and Savannah, Georgia, United States of America, and optimizes trapping sites to prevent transport, interdict and conduct surveillance for invading species.

\section{Materials and methods}

\subsection{Collection and control}

Mosquitoes were collected at Porto Cortes using CDC light traps baited with $\mathrm{CO} 2$ and octanol from October through December 2011. The efficacy of Entobac ${ }^{\mathrm{TM}}$ (Bacillus thuringiensis israelensis Bti biopesticide) delivered in the ProVector Flower Netty $\mathcal{Q}^{\mathrm{rM}}$ devices to control mosquitoes was tested against a control site in Porto Cortes. Entobac is an ecofriendly pesticide, that is non-toxic to humans, and is the first pesticide that has been developed to kill both larvae and adult mosquitoes (Yalwala et al. 2016). These devices were strategically hung in and around shelters and trees to reduce mosquito populations and provide the 
same area of coverage at a reduction of pesticide application approaching 10,000 times. Adult mosquitoes are attracted to the devices by the various colors and then probe the devices whereupon they ingest the Bti or the Bti attaches to the body of the mosquito. Adult male and female mosquitoes disseminate the Bti then die in about 7 days and when they land in water they kill the larvae mosquitoes as well at a rate of 3 larvae for each adult.

\subsection{Data analysis}

The Bioagent Transport and Environmental Modeling system (BioTEMS) was used to determine which vector mosquito species would most likely originate from Porto Cortez and possibly invade Savannah and Dubai. BioTEMS has been used to assist U.S. government and other agencies in predicting risk and countermeasures to biological weapons agents and infectious diseases. BioTEMS has also been used in conjunction with the Hazard Prediction Assessment Capabilities (HPAC) software to determine dispersal, persistence and reaerosolization during military and civilian exercise, assessing military facilities, and during presidential inaugurations. Input into BioTEMS modeling system included, mosquito species, biotic and abiotic factors, $\mathrm{n}$-dimensional hypervolume and TIGER phases. The TIGER model was developed to determine the phases of mosquito invasion; T-transport, I-introduction, G-gap infiltration, E-escalade, and R-residence \& recruitment. BioTEMS and ArcView were used to produce output into Google Earth.

\section{Results and discussion}

The following mosquito species were collected in Porto Cortes or have been recorded at the Walter Reed Biosystematics Unit at the Smithsonian Institute; Deinocerites cancer, Culex corniger, Culex nigripalpus, Haemagogus aeritinctus, Aedes angustivittatus, Aedes scapularis, Psorophora albipes, Wyeomyia celaenocephala, Aedes taeniorhynchus, Anopheles albimanus, Coquillettidia nigricans, Culex latisquama, Culex coronator, Culex quinquefasciatus, Uranotaenia lowii, Aedes hastatus, Mansonia titillans, Aedes albopictus, Psorophora ferox, and Uranotaenia orthodoxa. There was a significant reduction in mosquitoes at the test site verses the control site after 3 months of treatment using Bti delivered by devices (Mann Whitney $\mathrm{U}$ test; $\mathrm{Z}=-2.813, \mathrm{P}<0.01$ ). Using devices to deliver biopesticides can reduce the cost of pesticide application by as much as $1,000 \%$ while reducing non-target species exposure and environmental contamination. Optimal device placement at Porto Cortes, the port of origin, is shown in Figure 1.

The BioTEMS invasive species model identified four mosquito species as the main threat for invasion or reinvasion into the port of Savannah; Ae. albopictus, $\mathrm{Cu}$. coronator, and $\mathrm{M}$. titillans. These three species are vectors of several human and animal pathogens. The BioTEMS invasive species model was accurate in predicting invasion by $C$. coronator and $M$. titillans as they are in fact recent invaders into Savannah/Chatham County, Georgia (Moulis et al., 2008; Moulis et al. 2015). Although other species in Porto Cortes are also residents of the Savannah area they are less likely to reinvade due to physiological and environmental constraints. The threat area and optimal trap placement for surveillance within the immediate vicinity of the Savannah Port is shown in Figure 2.

The Jebel Ali port area presents a more hostile environment for invading species than the Savannah port, although areas in Dubai near the port are susceptible to invasion by several mosquito species being transported from Porto Cortes and Savannah, the most likely invading species are Ae. albopictus, Ae. vexans, and Cu. coronator. Other ports in Dubai may also provide a suitable means of introduction of invasive species through cruise and other ships coming into port. Optimal placements for some of these port areas are shown in Figure 3.

These four invasive species may constitute a significant health risk if transported by ship. In addition, reinvasion of infected mosquito species may reintroduce or bring in new pathogens into resident mosquito populations in the port area and surrounding city. These pathogens include zoonotic and transovarially transmitted pathogens such as dengue, West Nile and Zika viruses. Ae. albopictus poses a significant health risk from Honduras as it is a known vector of dengue, Chikungunya and Zika viruses and perhaps 21 other viruses (worldwide). Ae. vexans is a vector of West Nile virus, Rift Valley fever virus, and Dirofilaria immitis. Based upon the plasticity of Zika virus, and ability of $A$ e. vexans to vector Flaviviridae, Ae. vexans should be suspect of being a competent vector of Zika virus. $\mathrm{Cu}$. coronator is a potential vector of several arboviruses and has been found infected with Venezuelan equine encephalitis, West Nile virus, Saint Louis encephalitis virus. Mansonia titillans is a vector of Venezuelan equine encephalitis and filariasis. The receiving ports should also implement surveillance systems to ensure adequate coverage to sample port areas should these and other invasive mosquito species be introduced, including resting and breeding habitats. Continual surveillance utilizing several collection methods should be used concurrently, e.g. BG Sentinel, light and gravid traps, landing counts, sentinel animals and larvae collection. Connecting ports can reduce their risk to transporting and receiving invasive mosquito species and pathogens by implementing integrated mosquito management measures.

International shipping plays a significant role in the transport of medically important mosquito species. Vector control measures should include using economic and eco-friendly control of mosquito populations. Integrated mosquito management can be used to identify and reduce the risk of invasive mosquito species and their pathogens from other connected ports to Porto Cortes, Honduras, Savannah Port, USA and Jebel Ali, UAE. Additional risk analyses of biological agents and other vector-borne diseases for these port areas are being conducted, using lagrangian modeling and BioTEMS, including; anthrax, tularemia, and plague. Identifying the risk, conducting planning and expanding environmental surveillance and preparing medical countermeasures are essential should an accidental, criminal or terrorist release occur. The BioTEMS TIGER model provides information that can be utilized to interdict and control invasive mosquito species to reduce the risk they become permanently established. Port authorities have an opportunity to collaborate and coordinate mosquito control efforts with sister ports. Collaborative integrated mosquito management between ports not only reduces the risk of transporting and introducing invasive species but provides an important service to residents around the ports of origin who will benefit from reduced mosquito populations and reduced prevalence of vector-borne diseases.

\section{Acknowledgements}

The views expressed in this publication are those of the authors and do not reflect the official policy of the United States Army or United States Government. 


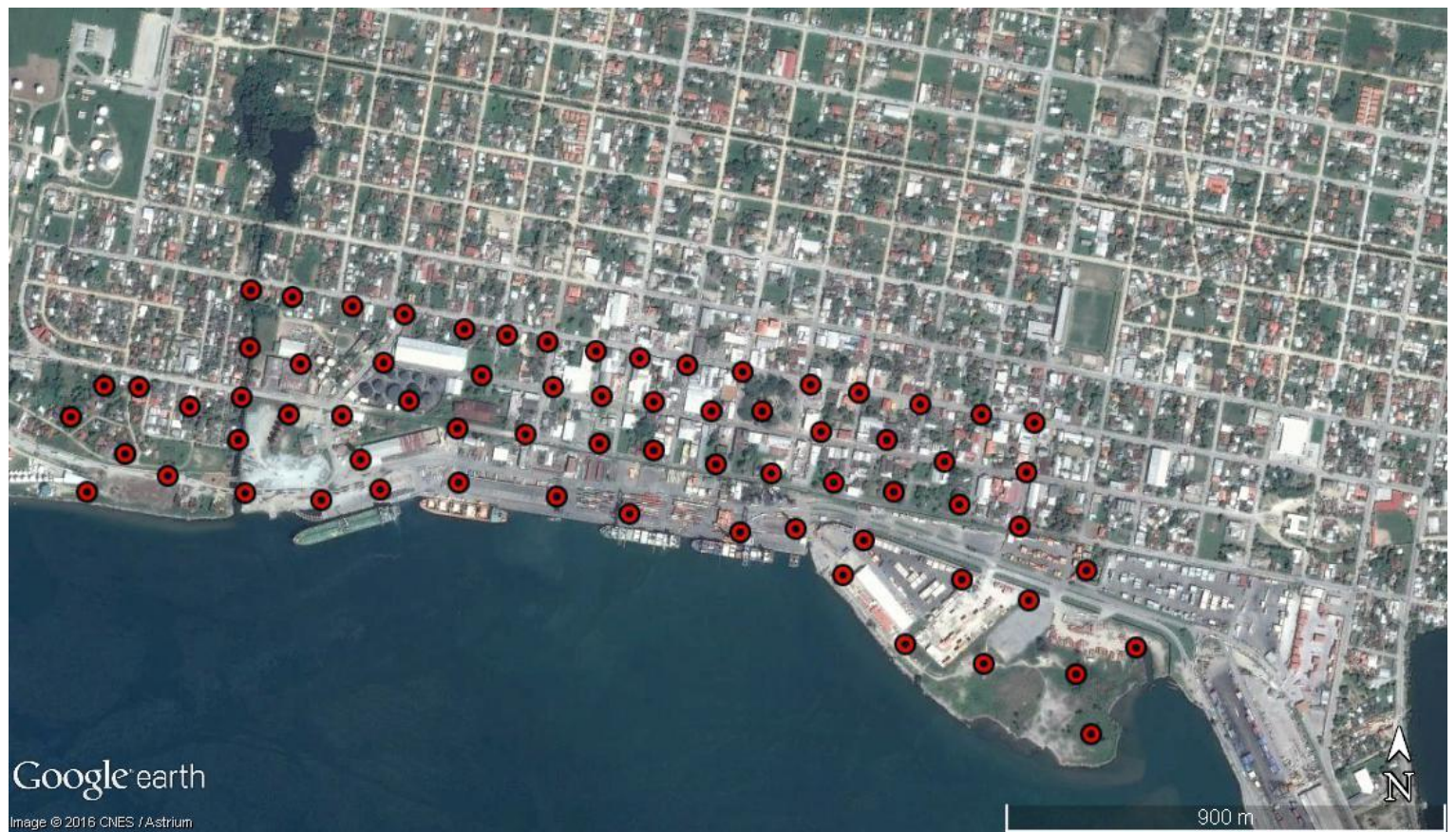

Fig. 1: Optimal Device Placement at the Container Ship Port of Origin in Porto Cortes, Honduras (Red Circles).

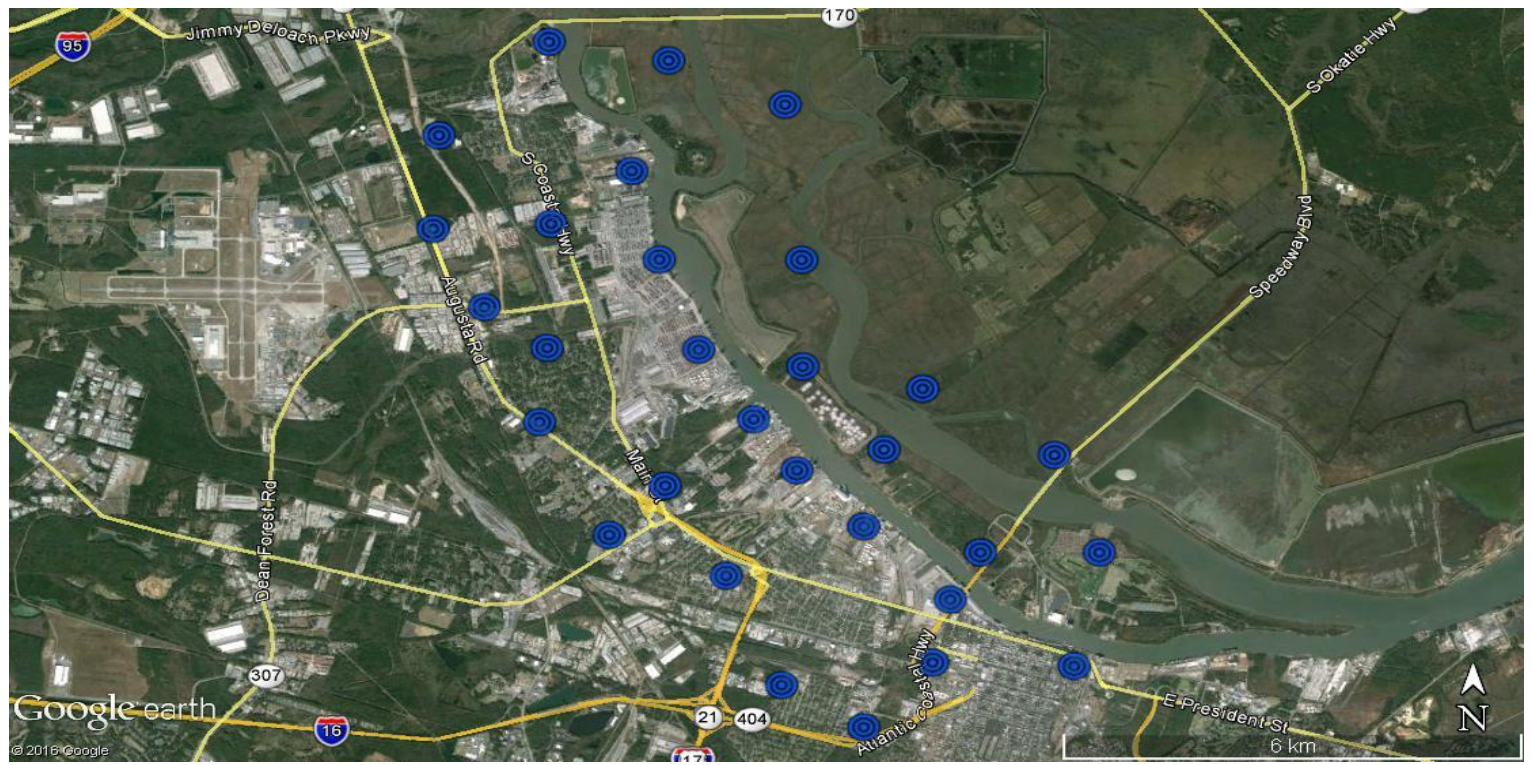

Fig. 2: Area of Optimal Trap Placement for Mosquito Surveillance within the Immediate Vicinity of the Savannah Port, United States of America (Blue Circles). 


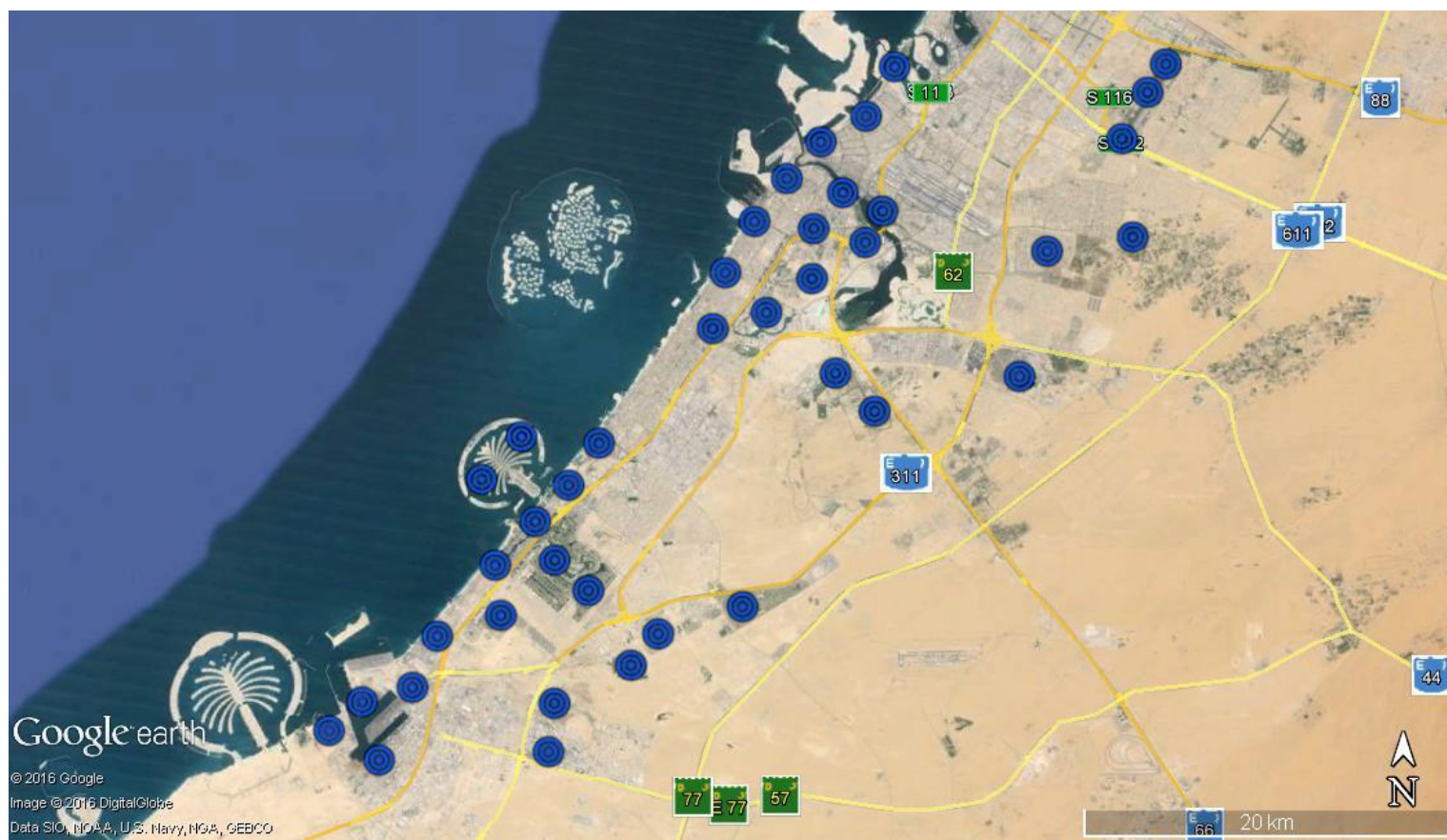

Fig. 3: Optimal Placements of Mosquito Surveillance in and Around Port Areas of Jabel Ali Port, United Arab Emirates (Blue Circles).

\section{References}

[1] Dar FK, Bayoumi R, al Karmi T, Shalabi A, Beidas F, Hussein MM (1993) Status of imported malaria in a control zone of the United Arab Emirates bordering an area of unstable malaria. Transactions Royal Society Tropical Medicine Hygiene 87:617-9. http://dx.doi.org/10.1016/0035-9203(93)90261-N.

[2] Inter-Press News Agency (2006) Anti-malaria drive keeps dengue at bay, Emirates News Agency. http://www.ipsnotizie.it/wam_en/news.php.

[3] Lodge D (2006) six degrees of separation? Trade globalization and changing linkages among freshwater ecosystems. Ecology in an era of globalization: challenges and opportunities for environmental scientists in the Americas. Proceedings of the Ecological Society of America International Conference.

[4] Meyerson L \& Mooney H (2007) Invasive Alien Species in an Era of Globalization. Frontiers in Ecology and the Environment 5:199208. http://dx doi.org/10./ 9295(2007)5[199:IASIAE]2.0.CO;2.

[5] Moulis RA, Peaty LF, Heusel JL, Lewandowski HB Jr, Harrison BA, Kelly R, \& Hager EJ. (2015). Mansonia titillans: New Resident Species or Infrequent Visitor in Chatham County, Georgia, and Beaufort County, South Carolina, USA. Journal American Mosqutio Control Association 31:167-71. http://dx.doi.org/10.2987/156491R.

[6] Moulis RA, Russell JD, Lewandowski HB Jr, Thompson PS, \& Heusel JL. (2008) Culex coronator in coastal Georgia and South Carolina. Journal American Mosqutio Control Association 24:58890. http://dx.doi.org/10.2987/5766.1.

[7] Tatem AJ, SI Hay SI, \& Rogers DJ (2006) Global traffic and disease vectors. Proceedings National Academy Sciences USA 103 6242-47. http://dx.doi.org/10.1073/pnas.0508391103.

[8] Yalwala S, Kollars JW, Kasembeli G, Barasa C, Senessie C, Kollars PG, Kollars TM Jr. (2016) Preliminary Report on the Reduction of Adult Mosquitoes in Housing Compounds in Western Kenya Using the ProVector Flower and Entobac Bait Pads Containing Bacillus thuringiensis israelensis With Honey Bait. Journal of Medical Entomology 53: 1242-1244. http://dx.doi.org/10.1093/jme/tjw075. 